\title{
Parastomal Hernia with Incarcerated Gallbladder: A Case Report
}

\author{
Ramon A Brown ${ }^{1 *}$, Brian Kann ${ }^{1}$ and James Woolridge ${ }^{2}$ \\ ${ }^{1}$ Department of Colon and Rectal Surgery, Ochsner Clinic Foundation, USA \\ ${ }^{2}$ Department of General Surgery, Ochsner Clinic Foundation, USA
}

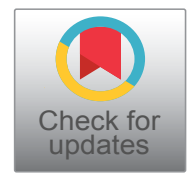

*Corresponding author: Ramon A Brown, MD, Department of Colon and Rectal Surgery, Ochsner Clinic Foundation, 1514 Jefferson Hwy, Jefferson, LA 70121, USA, Tel: 813-728-0768, E-mail: rbrown44@gmail.com

\begin{abstract}
Background: Parastomal hernias can occur in up to $37.8 \%$ of patients following an enterostomy [1]. This type of hernia develops through an intentional fascial defect. Commonly these hernias may contain a variety of contents including omentum, small bowel, or colon, but very rarely contains the gallbladder. Few cases are reported in the literature and guidance to the appropriate management of these patients is managed on a case by case basis.
\end{abstract}

Case presentation: We report the presentation and management of an 83-year-old Caucasian female with a gallbladder hernia into a parastomal defect and outline the operative management.

Conclusions: Incarcerated parastomal hernia involving the gallbladder is rare entity, best diagnosed by CT scan. Management in the case of this patient involved operative reduction, cholecystectomy, consideration of closure of enterostomy and of repair of the fascial defect.

\section{Background}

A stoma is a surgically created opening on the outer surface of the abdomen, through which urine or feces can be diverted into an external pouch following removal of part of the bowel or urinary system. Parastomal hernias can occur weeks or years after stoma placement as consequence the creation of an abdominal wall defect [2]. The contents of these hernias commonly include the omentum, small bowel and colon; rarely, the hernia sac may contain a variety of other intra-abdominal structures including the gallbladder. The utilization of computerized tomography scans has facilitated the identification of the contents of these hernias, the presence of which have implications for definitive operative management. An incarcerated gallbladder hernia is a rare condition, and a small number of case reports describe the process of diagnosis and subsequent management [3-5]. This case report outlines the presentation, diagnosis and operative management of one such patient.

\section{Case Presentation}

A 63-year-old white female with a complicated history following surgery for diverticular bleeding is referred for elective colostomy reversal. She had initially undergone a left colectomy with a distal transverse colon to sigmoid colon anastomosis; this was complicated by an anastomotic leak and left upper quadrant abscess, necessitating distal transverse colectomy and splenectomy, with creation of a right upper quadrant end transverse colostomy. A long Hartmann's pouch consisting of rectum and sigmoid colon was left in situ. At initial presentation, she complained of a large peristomal bulge and associated abdominal bloating. She denied abdominal pain with eating, nausea, or vomiting. Colonoscopy revealed only $18 \mathrm{~cm}$ of colon remaining from the colostomy to the cecum; the residual rectosigmoid stump measured $55 \mathrm{~cm}$ in length and was characterized by diffuse diverticular disease. A preoperative CT scan of the abdomen and pelvis demonstrated a large parastomal hernia with incarceration of the small bowel and gallbladder, as well as a midline fascial defect (Figure 1 and Figure 2). She subsequently underwent colostomy takedown with completion sigmoid colectomy, derotation of the right colon with an ascending colon-rectal anastomosis, cholecystectomy, appendectomy, and abdominal wall reconstruction at both the ostomy site and midline defect with on-lay bioprosthetic mesh. She had an uncomplicated post-operative course, and upon outpatient follow-up, she reported excellent bowel function without hernia recurrence.

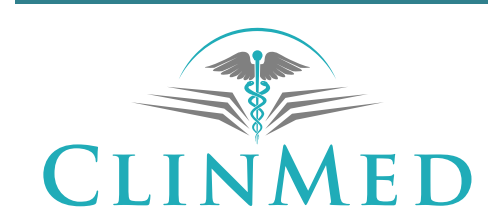

INTERNATIONAL LIBRARY

Citation: Brown RA, Kann B, Woolridge J (2018) Parastomal Hernia with Incarcerated Gallbladder: A Case Report. Int J Surg Res Pract 5:072. doi.org/10.23937/2378-3397/1410072 Received: April 07, 2018: Accepted: May 21, 2018; Published: May 23, 2018

Copyright: (c) 2018 Brown RA, et al. This is an open-access article distributed under the terms of the Creative Commons Attribution License, which permits unrestricted use, distribution, and reproduction in any medium, provided the original author and source are credited. 

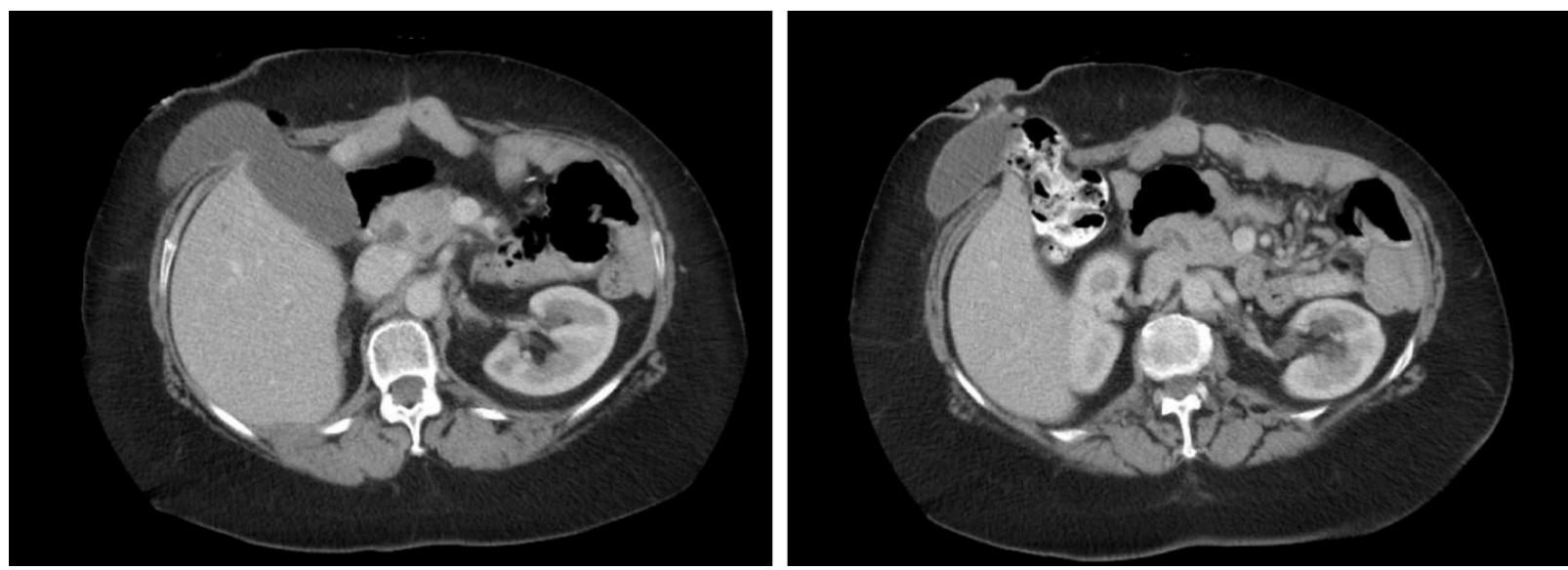

Figure 1: $a, b)$ Axial CT imaging showing herniation of gall bladder through a right upper quadrant parastomal hernia.
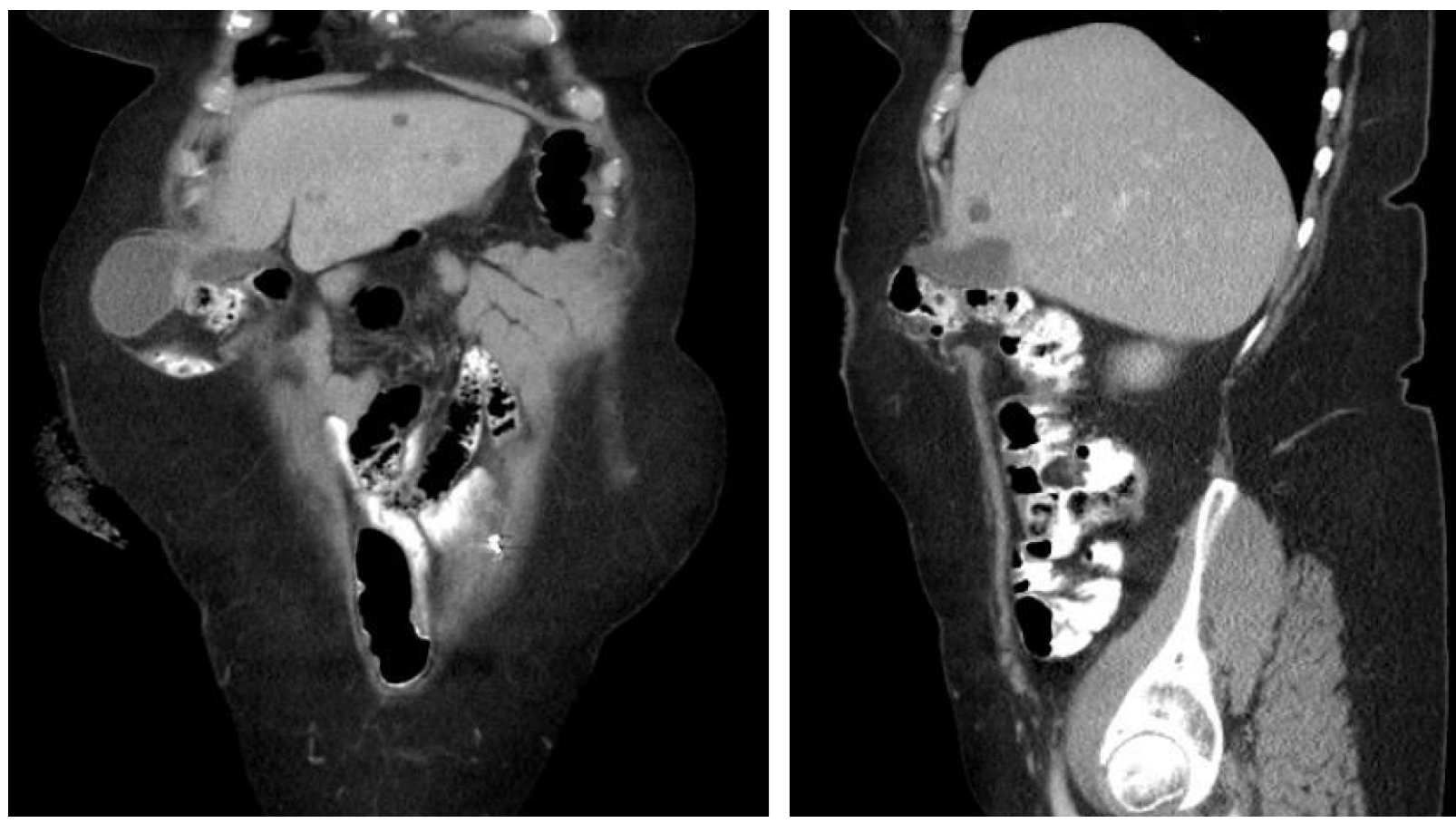

Figure 2: $a, b)$ Coronal and sagittal CT imaging showing herniation of gall bladder through a right upper quadrant parastomal hernia.

\section{Discussion}

Herniation and incarceration of the gallbladder in a parastomal hernia is an uncommon event and few cases have been reported in the literature [2,5-7]. A recent case report identified patterns of presentation and management. The majority of reported patients were of older age, female and had a propensity to the development of other ventral hernias, especially incisional hernias.

The utilization of CT imaging is the modality of choice in the diagnosis of this disease entity. Identification of the gallbladder within the hernia sac aids in the guidance of operative planning as the resection is likely necessary to achieve abdominal wall closure and prevention of recurrence. In our case careful evaluation for colostomy closure with resection of the diseased segment sigmoid colon and derotation of the right colon facilitated abdominal wall reconstruction by allowing closure of the all abdominal wall defects. Abdominal wall reconstruction with bioprosthetic mesh reduces recurrence of herniation at the midline incisional hernia site and the trephination. Cholecystectomy and abdominal wall reconstruction has been described similarly on case reports [5].

\section{Conclusion}

In summary, gall bladder herniation through the abdominal wall in a parastomal hernia is a rare entity. Case reports on the topic have proposed similar methods of management; however, in our case colostomy reversal facilitated abdominal wall closure at all sites without morbidity or mortality. Management of these cases deserve careful and individualized attention with reversal of enterostomy performed when possible.

\section{Disclaimer}

The authors have nothing to disclose. No financial support was provided for this submission. The views ex- 
pressed in this article are those of the authors, and do not reflect the official policy or position of the Ochsner Clinic Foundation or any of its affiliates.

\section{Authorship}

The authors listed contributed to this manuscript through the conception, design, acquisition of data, analysis and interpretation of data as well as drafting the article and revising it critically for important intellectual content. Brian Kann, MD and James Woolridge, MD gave final approval of the version to be published.

\section{References}

1. Sohn YJ, Moon SM, Shin US, Jee SH (2012) Incidence and risk factors of parastomal hernia. J Korean Soc Coloproctol 28: $241-246$.
2. Garcia RM, Brody F, Miller J, Ponsky TA (2005) Parastomal herniation of the gallbladder. Hernia 9: 397-399.

3. Aguirre DA, Santosa AC, Casola G, Sirlin CB (2005) Abdominal Wall Hernias: Imaging Features, Complications, and Diagnostic Pitfalls at Multi-Detector Row CT. RadioGraphics 25: 1501-1520.

4. Gomez Artacho M, Boisset G, Taoum C, Navarro F (2016) Complicated parastomal hernia as a clinical presentation of a gallbladder hydrops. Cir Esp 95: 347.

5. To H, Brough S, Pande G (2015) Case report and operative management of gallbladder herniation. BMC Surg 15: 72.

6. St Peter SD, Heppell J (2005) Surgical images: soft tissue: incarcerated gallbladder in a parastomal hernia. Can J Surg 48: 46.

7. Rashid M, Abayasekara K, Mitchell E (2010) A case report of an incarcerated gallbladder in a parastomal hernia. Int $J$ Surg 23: 8 . 